\title{
Factors Influencing Childbearing Decisions and Knowledge of Perinatal Risks among Canadian Men and Women
}

Suzanne Tough • Karen Tofflemire • Karen Benzies •

Nonie Fraser-Lee · Christine Newburn-Cook

Published online: 13 March 2007

(C) Springer Science+Business Media, LLC 2007

Erratum to: Maternal and Child Health Journal, Vol. 11, No. 2, March 2007. DOI: 10.1007/s10995-006-0156-1

In the above mentioned article, the second author (Karen Tofflemire) was not listed on the paper. 